\title{
Statistical rates in approximation by positive linear operators
}

Esra Erkus-Duman 


\title{
STATISTICAL RATES IN APPROXIMATION BY POSITIVE LINEAR OPERATORS
}

\author{
ESRA ERKUŞ-DUMAN
}

Received December 18, 2010

\begin{abstract}
This study is the continuation of our former work [O. Duman and E. Erkuş, Comput. Math. Appl. 52 (2006) 967-974] in which we obtained a statistical Korovkin-type approximation theorem for a sequence of positive linear operators defined on the space of all real-valued continuous and $2 \pi$ periodic functions on the real $m$-dimensional space. In this paper, we compute the statistical rates of this statistical approximation.
\end{abstract}

2000 Mathematics Subject Classification: 41A25; 41A36

Keywords: statistical convergence, statistical rates, positive linear operators, periodic functions, modulus of continuity

\section{INTRODUCTION}

The motivation of this work are [3,4]. Let $m$ be a positive integer, and let $C^{*}\left(\mathbb{R}^{m}\right)$ denote the space of all real-valued continuous and $2 \pi$ periodic functions on the real $m$-dimensional space $\mathbb{R}^{m}$. Here, the $2 \pi$-periodicitiy of a function $f \in C^{*}\left(\mathbb{R}^{m}\right)$ is given by

$$
\begin{aligned}
f\left(u_{1}, u_{2}, \ldots, u_{m}\right)= & f\left(u_{1}+2 k \pi, u_{2}, \ldots, u_{m}\right) \\
= & f\left(u_{1}, u_{2}+2 k \pi, \ldots, u_{m}\right) \\
& \ldots \\
= & f\left(u_{1}, u_{2}, \ldots, u_{m}+2 k \pi\right)
\end{aligned}
$$

for every $\mathbf{u}=\left(u_{1}, u_{2}, \ldots, u_{m}\right) \in \mathbb{R}^{m}$ and $k=0, \pm 1, \ldots$ (see, for instance, [10, p. 126]). Consider the usual supremum norm on $C^{*}\left(\mathbb{R}^{m}\right)$ defined by

$$
\|f\|_{*}:=\sup _{\left(u_{1}, u_{2}, \ldots, u_{m}\right) \in \mathbb{R}^{m}}\left|f\left(u_{1}, u_{2}, \ldots, u_{m}\right)\right|, \quad f \in C^{*}\left(\mathbb{R}^{m}\right) .
$$

Assume that

$$
A:=\left[a_{j n}\right] \quad(j, n \in \mathbb{N}:=\{1,2, \ldots\})
$$


is a non-negative summability matrix. Recently, in [4], it has been proved that, for any sequence $\left\{L_{n}\right\}$ of positive linear operators mapping $C^{*}\left(\mathbb{R}^{m}\right)$ into itself,

$$
s t_{A}-\lim _{n}\left\|L_{n}(f)-f\right\|_{*}=0 \text { for all } f \in C^{*}\left(\mathbb{R}^{m}\right)
$$

if and only if

$$
s t_{A}-\lim _{n}\left\|L_{n}\left(f_{i}\right)-f_{i}\right\|_{*}=0 \text { for each } i=0,1, \ldots, 2 m+1,
$$

where

$$
\begin{aligned}
& f_{0}\left(u_{1}, \ldots, u_{m}\right)=1 \\
& f_{i}\left(u_{1}, \ldots, u_{m}\right)=\cos u_{i} \text { for } i=1,2, \ldots, m, \\
& f_{j}\left(u_{1}, \ldots, u_{m}\right)=\sin u_{j} \text { for } j=m+1, m+2, \ldots, 2 m .
\end{aligned}
$$

Notice that by $s t_{A}-\lim _{n} x_{n}=L$ we mean that the sequence $x:=\left\{x_{n}\right\}$ is $A$-statistically convergent to $L$ (see [8]), i.e., for every $\varepsilon>0$,

$$
\lim _{j} \sum_{n:\left|x_{n}-L\right| \geq \varepsilon} a_{j n}=0
$$

It is well-known that every convergent sequence is $A$-statistically convergent to the same value, however the converse is not always true. Also, taking special regular matrices, one can obtain many convergence methods from the $A$-statistical convergence. For example, if $A=C_{1}$, the Cesáro matrix, then it reduces to the concept of statistical convergence (see [7,9]), and if $A=I$, the identity matrix, then it coincides with the ordinary convergence. Hence, with such properties, the usage of $A$-statistical convergence in approximation theory provides us more powerful results than the classical theory does. Observe that the above statistical approximation theorem in the space $C^{*}\left(\mathbb{R}^{m}\right)$ contains the classical uniform convergence. However, it is also possible to construct a sequence of positive linear operators satisfying (1.1) or (1.2) but not the corresponding classical case (see [4]).

In this paper, we mainly discuss the following problem: how can we compute the statistical rates of the $A$-statistical convergence to zero of the difference sequence $\left\{\left\|L_{n}(f)-f\right\|_{*}\right\}$ in (1.1)? Such a problem has recently been investigated for the one dimensional case by Duman [3]. In order to compute the statistical rates we use the following two definitions (see, for instance, $[5,6]$ ):

Let $\left\{p_{n}\right\}$ be a positive non-increasing sequence of real numbers. For a given sequence $\left\{x_{n}\right\}$, we say that

- $\left\{x_{n}\right\}$ is $A$-statistically convergent to the number $L$ with rate $o\left(p_{n}\right)$, denoted by $x_{n}-L=s t_{A}-o\left(p_{n}\right)$ as $n \rightarrow \infty$, if, for every $\varepsilon>0$,

$$
\lim _{j} \frac{1}{p_{j}} \sum_{n:\left|x_{n}-L\right| \geq \varepsilon} a_{j n}=0,
$$


- $\left\{x_{n}\right\}$ is $A$-statistically convergent to the number $L$ with rate $o_{m}\left(p_{n}\right)$, denoted by $x_{n}-L=s t_{A}-o_{m}\left(p_{n}\right)$ as $n \rightarrow \infty$, if, for every $\varepsilon>0$,

$$
\lim _{j} \sum_{n:\left|x_{n}-L\right| \geq \varepsilon p_{n}} a_{j n}=0 .
$$

Notice that the rate of convergence given by the little $o$ is influenced more strongly by the summability method then by the terms of the sequence $\left\{x_{n}\right\}$. For instance, when one takes the identity matrix $I$, if $\left\{p_{n}\right\}$ is any positive non-increasing sequence satisfying the following inequality:

$$
\frac{1}{p_{n}} \leq M \quad(\text { for } M>0 \text { and } n \in \mathbb{N}),
$$

then we have $x_{n}-L=s t_{A}-o\left(p_{n}\right)$ as $n \rightarrow \infty$ for any convergent sequence $\left\{x_{n}-L\right\}$ regardless of how slowly it goes to zero. To avoid such an unfortunate situation, one may borrow the concept of convergence in measure from the measure theory to define the rate of convergence as in the notion of the little $o_{m}$.

\section{STATISTICAL RATES OF THE APPROXIMATION}

In this section, we compute the statistical rates of the statistical approximation in (1.1). To see this we first recall the concept of modulus of continuity in the space $C^{*}\left(\mathbb{R}^{m}\right)$. The modulus of continuity of a function $f$ belonging to $C^{*}\left(\mathbb{R}^{m}\right)$ is given by, for any $\delta>0$,

$$
w(f, \delta)=\sup _{\sqrt{\left(u_{1}-x_{1}\right)^{2}+\ldots+\left(u_{m}-x_{m}\right)^{2}} \leq \delta}\left|f\left(u_{1}, \ldots, u_{m}\right)-f\left(x_{1}, \ldots, x_{m}\right)\right| .
$$

It is well-known that a necessary and sufficient condition for a function $f$ to belong to $C^{*}\left(\mathbb{R}^{m}\right)$ is $\lim _{\delta \rightarrow 0} w(f, \delta)=0$ (see [1, p. 80]). Furthermore, it follows from (2.1) that, for every $f \in C^{*}\left(\mathbb{R}^{m}\right)$ and $\left(u_{1}, \ldots, u_{m}\right),\left(x_{1}, \ldots, x_{m}\right) \in \mathbb{R}^{m}$,

$$
\left|f\left(u_{1}, \ldots, u_{m}\right)-f\left(x_{1}, \ldots, x_{m}\right)\right| \leq w\left(f, \sqrt{\left(u_{1}-x_{1}\right)^{2}+\ldots+\left(u_{m}-x_{m}\right)^{2}}\right),
$$

and

$$
w(f, c \delta) \leq(1+c) w(f, \delta) \text { for any } c, \delta>0 .
$$

Now we are ready to state our main results.

Theorem 1. Let $A=\left[a_{j n}\right]$ be a non-negative regular summability matrix and let $\left\{L_{n}\right\}$ be a sequence of positive linear operators mapping $C^{*}\left(\mathbb{R}^{m}\right)$ into itself. For each $\left(x_{1}, \ldots, x_{m}\right) \in \mathbb{R}^{m}$, define the function $\varphi_{x_{1}, \ldots, x_{m}}$ by

$$
\varphi_{x_{1}, \ldots, x_{m}}\left(u_{1}, \ldots, u_{m}\right)=\sin ^{2}\left(\frac{u_{1}-x_{1}}{2}\right)+\ldots+\sin ^{2}\left(\frac{u_{m}-x_{m}}{2}\right) .
$$

Assume that $\left\{p_{n}\right\}$ and $\left\{q_{n}\right\}$ are positive non-increasing sequences of real numbers. If, for every $f \in C^{*}\left(\mathbb{R}^{m}\right)$, the conditions 
(i) $\left\|L_{n}\left(f_{0}\right)-f_{0}\right\|_{*}=s t_{A}-o\left(p_{n}\right)$ as $n \rightarrow \infty$ with $f_{0}\left(u_{1}, \ldots, u_{m}\right)=1$,

(ii) $w(f, \delta)=s t_{A}-o\left(q_{n}\right)$ as $n \rightarrow \infty$ with $\delta_{n}:=\sqrt{\left\|L_{n}\left(\varphi_{x_{1}, \ldots, x_{m}}\right)\right\|_{*}}$

hold, then we have

$$
\left\|L_{n}(f)-f\right\|_{*}=s t_{A}-o\left(r_{n}\right) \text { as } n \rightarrow \infty
$$

with $r_{n}:=\max \left\{p_{n}, q_{n}\right\}$ for each $n \in \mathbb{N}$.

Proof. Let $f \in C^{*}\left(\mathbb{R}^{m}\right)$ and $\left(x_{1}, \ldots, x_{m}\right) \in \mathbb{K}:=[-\pi, \pi] \times \ldots \times[-\pi, \pi]$ be fixed. Since

$$
\begin{aligned}
& \left|L_{n}\left(f ; x_{1}, \ldots, x_{m}\right)-f\left(x_{1}, \ldots, x_{m}\right)\right| \\
\leq & L_{n}\left(\left|f\left(u_{1}, \ldots, u_{m}\right)-f\left(x_{1}, \ldots, x_{m}\right)\right| ; x_{1}, \ldots, x_{m}\right) \\
& +\left|f\left(x_{1}, \ldots, x_{m}\right)\right|\left|L_{n}\left(f_{0} ; x_{1}, \ldots, x_{m}\right)-f_{0}\left(x_{1}, \ldots, x_{m}\right)\right|,
\end{aligned}
$$

it follows from (2.2) that

$$
\begin{aligned}
& \left|L_{n}\left(f ; x_{1}, \ldots, x_{m}\right)-f\left(x_{1}, \ldots, x_{m}\right)\right| \\
\leq & L_{n}\left(w\left(f, \sqrt{\left(u_{1}-x_{1}\right)^{2}+\ldots+\left(u_{m}-x_{m}\right)^{2}}\right) ; x_{1}, \ldots, x_{m}\right) \\
& +M\left|L_{n}\left(f_{0} ; x_{1}, \ldots, x_{m}\right)-f_{0}\left(x_{1}, \ldots, x_{m}\right)\right|,
\end{aligned}
$$

where $M:=\|f\|_{*}$. Since, for all $t \in[-\pi, \pi]$,

$$
|t| \leq \pi\left|\sin \frac{t}{2}\right|
$$

we have

$$
\sqrt{\left(u_{1}-x_{1}\right)^{2}+\ldots+\left(u_{m}-x_{m}\right)^{2}} \leq \pi \sqrt{\varphi_{x_{1}, \ldots, x_{m}}\left(u_{1}, \ldots, u_{m}\right)},
$$

where $\varphi_{x_{1}, \ldots, x_{m}}$ is given by (2.4). Combining this with (2.5), we obtain that

$$
\begin{gathered}
\left|L_{n}\left(f ; x_{1}, \ldots, x_{m}\right)-f\left(x_{1}, \ldots, x_{m}\right)\right| \leq L_{n}\left(w\left(f, \pi \sqrt{\varphi_{x_{1}}, \ldots, x_{m}}\right) ; x_{1}, \ldots, x_{m}\right) \\
+M\left|L_{n}\left(f_{0} ; x_{1}, \ldots, x_{m}\right)-f_{0}\left(x_{1}, \ldots, x_{m}\right)\right| .
\end{gathered}
$$

Also, using (2.3), we may write that, for any $\delta>0$,

$$
\begin{aligned}
\mid L_{n}\left(f ; x_{1}, \ldots, x_{m}\right)- & f\left(x_{1}, \ldots, x_{m}\right) \mid \leq w(f, \delta) L_{n}\left(1+\frac{\pi}{\delta} \sqrt{\varphi_{x_{1}, \ldots, x_{m}}} ; x_{1}, \ldots, x_{m}\right) \\
+ & M\left|L_{n}\left(f_{0} ; x_{1}, \ldots, x_{m}\right)-f_{0}\left(x_{1}, \ldots, x_{m}\right)\right| \\
& \leq w(f, \delta) L_{n}\left(f_{0} ; x_{1}, \ldots, x_{m}\right) \\
& +\frac{\pi w(f, \delta)}{\delta} L_{n}\left(\sqrt{\varphi_{x_{1}, \ldots, x_{m}}} ; x_{1}, \ldots, x_{m}\right) \\
+ & M\left|L_{n}\left(f_{0} ; x_{1}, \ldots, x_{m}\right)-f_{0}\left(x_{1}, \ldots, x_{m}\right)\right| .
\end{aligned}
$$

From the Cauchy-Schwarz inequality for positive linear operators (see [2]), we have

$$
\left|L_{n}\left(f ; x_{1}, \ldots, x_{m}\right)-f\left(x_{1}, \ldots, x_{m}\right)\right|
$$




$$
\begin{gathered}
\leq w(f, \delta)+w(f, \delta)\left|L_{n}\left(f_{0} ; x_{1}, \ldots, x_{m}\right)-f_{0}\left(x_{1}, \ldots, x_{m}\right)\right| \\
+\frac{\pi w(f, \delta)}{\delta} \sqrt{L_{n}\left(f_{0} ; x_{1}, \ldots, x_{m}\right)} \sqrt{L_{n}\left(\varphi_{x_{1}, \ldots, x_{m}} ; x_{1}, \ldots, x_{m}\right)} \\
\quad+M\left|L_{n}\left(f_{0} ; x_{1}, \ldots, x_{m}\right)-f_{0}\left(x_{1}, \ldots, x_{m}\right)\right| .
\end{gathered}
$$

Now taking supremum over $\left(x_{1}, \ldots, x_{m}\right) \in \mathbb{K}$ and choosing $\delta:=\delta_{n}=\sqrt{\left\|L_{n}\left(\varphi_{x_{1}, \ldots, x_{m}}\right)\right\|_{*}}$, we have

$$
\begin{aligned}
\left\|L_{n}(f)-f\right\|_{*} \leq & (1+\pi) w\left(f, \delta_{n}\right)+w\left(f, \delta_{n}\right)\left\|L_{n}\left(f_{0}\right)-f_{0}\right\|_{*} \\
& +\pi w\left(f, \delta_{n}\right) \sqrt{\left\|L_{n}\left(f_{0}\right)-f_{0}\right\|_{*}} \\
& +M\left\|L_{n}\left(f_{0}\right)-f_{0}\right\|_{*} .
\end{aligned}
$$

Letting $C:=\max \{1+\pi, M\}$, the last inequality gives that

$$
\begin{aligned}
\left\|L_{n}(f)-f\right\|_{*} & \leq C\left\{w\left(f, \delta_{n}\right)+w\left(f, \delta_{n}\right)\left\|L_{n}\left(f_{0}\right)-f_{0}\right\|_{*}\right. \\
& \left.+w\left(f, \delta_{n}\right) \sqrt{\left\|L_{n}\left(f_{0}\right)-f_{0}\right\|_{*}}+\left\|L_{n}\left(f_{0}\right)-f_{0}\right\|_{*}\right\} .
\end{aligned}
$$

Now, for a given $\varepsilon>0$, define the following sets:

$$
\begin{aligned}
D: & =\left\{n \in \mathbb{N}:\left\|L_{n}(f)-f\right\|_{*} \geq \varepsilon\right\}, \\
D_{1}: & =\left\{n \in \mathbb{N}:\left\|L_{n}\left(f_{0}\right)-f_{0}\right\|_{*} \geq \frac{\varepsilon}{4 C}\right\}, \\
D_{2}: & =\left\{n \in \mathbb{N}: w\left(f, \delta_{n}\right) \geq \frac{\varepsilon}{4 C}\right\}, \\
D_{3}: & =\left\{n \in \mathbb{N}: w\left(f, \delta_{n}\right)\left\|L_{n}\left(f_{0}\right)-f_{0}\right\|_{*} \geq \frac{\varepsilon}{4 C}\right\}, \\
D_{4}: & =\left\{n \in \mathbb{N}: w\left(f, \delta_{n}\right) \sqrt{\left\|L_{n}\left(f_{0}\right)-f_{0}\right\|_{*}} \geq \frac{\varepsilon}{4 C}\right\} .
\end{aligned}
$$

By (2.6) it is easy to that $D \subseteq \bigcup_{i=0}^{4} D_{i}$. Furthermore, consider the sets

$$
\begin{gathered}
D_{3}^{\prime}:=\left\{n \in \mathbb{N}: w\left(f, \delta_{n}\right) \geq \sqrt{\frac{\varepsilon}{4 C}}\right\}, \\
D_{3}^{\prime \prime}:=\left\{n \in \mathbb{N}:\left\|L_{n}\left(f_{0}\right)-f_{0}\right\|_{*} \geq \sqrt{\frac{\varepsilon}{4 C}}\right\} .
\end{gathered}
$$

Then observe that $D_{3} \subseteq D_{3}^{\prime} \cup D_{3}^{\prime \prime}$ and $D_{4} \subseteq D_{1} \cup D_{3}^{\prime \prime}$, so we have

$$
D \subseteq D_{1} \cup D_{2} \cup D_{3}^{\prime} \cup D_{3}^{\prime \prime} \text {. }
$$

This inclusion implies, for all $j \in \mathbb{N}$, that

$$
\frac{1}{r_{j}} \sum_{n \in D} a_{j n} \leq \frac{1}{p_{j}} \sum_{n \in D_{1}} a_{j n}+\frac{1}{q_{j}} \sum_{n \in D_{2}} a_{j n}+\frac{1}{q_{j}} \sum_{n \in D_{3}^{\prime}} a_{j n}+\frac{1}{p_{j}} \sum_{n \in D_{3}^{\prime \prime}} a_{j n} .
$$


Now taking limit as $j \rightarrow \infty$ in (2.7) and using the hypotheses $(i)$ and ( $i i)$, we conclude that

$$
\lim _{j} \frac{1}{r_{j}} \sum_{n \in D} a_{j n}=0
$$

which means

$$
\left\|L_{n}(f)-f\right\|_{*}=s t_{A}-o\left(r_{n}\right) \text { as } n \rightarrow \infty,
$$

so the theorem is proved.

In a similar manner, we get the next result, which involves the statistical rate with the little $o_{m}$.

Theorem 2. Let $A=\left[a_{j n}\right],\left\{L_{n}\right\},\left\{\delta_{n}\right\},\left\{p_{n}\right\}$ and $\left\{q_{n}\right\}$ be the same as in Theorem 1. Assume that the conditions ( $i$ ) and ( $i i)$ of Theorem 1 hold for the little $o_{m}$ instead of the little o. Then, for any $f \in C^{*}\left(\mathbb{R}^{m}\right)$, we have

$$
\left\|L_{n}(f)-f\right\|_{*}=s t_{A}-o_{m}\left(s_{n}\right) \text { as } n \rightarrow \infty
$$

with $s_{n}:=\max \left\{p_{n}, q_{n}, \sqrt{p_{n}}, p_{n} q_{n}\right\}$ for each $n \in \mathbb{N}$.

Proof. Since $s_{n}=\max \left\{p_{n}, q_{n}, \sqrt{p_{n}}, p_{n} q_{n}\right\}$, from (2.6), we immediately get that

$$
\begin{aligned}
\frac{1}{s_{n}}\left\|L_{n}(f)-f\right\|_{*} & \leq C\left\{\frac{1}{q_{n}} w\left(f, \delta_{n}\right)+\frac{1}{p_{n} q_{n}} w\left(f, \delta_{n}\right)\left\|L_{n}\left(f_{0}\right)-f_{0}\right\|_{*}\right. \\
& \left.+\frac{1}{q_{n}} w\left(f, \delta_{n}\right) \sqrt{\frac{1}{p_{n}}\left\|L_{n}\left(f_{0}\right)-f_{0}\right\|_{*}}+\frac{1}{p_{n}}\left\|L_{n}\left(f_{0}\right)-f_{0}\right\|_{*}\right\} .
\end{aligned}
$$

Then, for a given $\varepsilon>0$, consider the following sets:

$$
\begin{aligned}
E: & =\left\{n \in \mathbb{N}:\left\|L_{n}(f)-f\right\|_{*} \geq \varepsilon s_{n}\right\}, \\
E_{1}: & =\left\{n \in \mathbb{N}:\left\|L_{n}\left(f_{0}\right)-f_{0}\right\|_{*} \geq \frac{\varepsilon p_{n}}{4 C}\right\}, \\
E_{2}: & =\left\{n \in \mathbb{N}: w\left(f, \delta_{n}\right) \geq \frac{\varepsilon q_{n}}{4 C}\right\}, \\
E_{3}: & =\left\{n \in \mathbb{N}: \frac{w\left(f, \delta_{n}\right)}{q_{n}} \frac{\left\|L_{n}\left(f_{0}\right)-f_{0}\right\|_{*}}{p_{n}} \geq \frac{\varepsilon}{4 C}\right\}, \\
E_{4}: & =\left\{n \in \mathbb{N}: \frac{w\left(f, \delta_{n}\right)}{q_{n}} \sqrt{\frac{\left\|L_{n}\left(f_{0}\right)-f_{0}\right\|_{*}}{p_{n}}} \geq \frac{\varepsilon}{4 C}\right\} .
\end{aligned}
$$

In this case, it follows from (2.8) that

$$
E \subseteq \bigcup_{i=1}^{4} E_{i} .
$$


Also letting

$$
\begin{aligned}
& E_{3}^{\prime}:=\left\{n \in \mathbb{N}: w\left(f, \delta_{n}\right) \geq q_{n} \sqrt{\frac{\varepsilon}{4 C}}\right\}, \\
& E_{3}^{\prime \prime}:=\left\{n \in \mathbb{N}:\left\|L_{n}\left(f_{0}\right)-f_{0}\right\|_{*} \geq p_{n} \sqrt{\frac{\varepsilon}{4 C}}\right\},
\end{aligned}
$$

we get $E_{3} \subseteq E_{3}^{\prime} \cup E_{3}^{\prime \prime}$ and $E_{4} \subseteq E_{1} \cup E_{3}^{\prime \prime}$, which implies

$$
E \subseteq E_{1} \cup E_{2} \cup E_{3}^{\prime} \cup E_{3}^{\prime \prime} \text {. }
$$

Hence, the last inclusion yields that, for every $j \in \mathbb{N}$,

$$
\sum_{n \in E} a_{j n} \leq \sum_{n \in E_{1}} a_{j n}+\sum_{n \in E_{2}} a_{j n}+\sum_{n \in E_{3}^{\prime}} a_{j n}+\sum_{n \in E_{3}^{\prime \prime}} a_{j n} .
$$

Now taking limit as $j \rightarrow \infty$ and using the hypotheses, we immediately see that

$$
\lim _{j} \sum_{n \in E} a_{j n}=0
$$

whence the result is proved.

Remark 1. Specializing the sequences $\left\{p_{n}\right\}$ and $\left\{q_{n}\right\}$ as $p_{n}=q_{n}=1$ for each $n \in \mathbb{N}$, Theorem 2.3 of [4] is a special case of our Theorem 1 or Theorem 2. So, our results give us the statistical rates of approximation to a function $f \in C^{*}\left(\mathbb{R}^{m}\right)$ by positive linear operators mapping $C^{*}\left(\mathbb{R}^{m}\right)$ into itself.

\section{REFERENCES}

[1] G. A. Anastassiou and S. G. Gal, Approximation theory. Moduli of continuity and global smoothness preservation. Boston, MA: Birkhäuser Boston, Inc., 2000.

[2] R. A. DeVore, The approximation of continuous functions by positive linear operators, ser. Lecture Notes in Mathematics. Berlin-New York: Springer-Verlag, 1972, vol. 293.

[3] O. Duman, "Regular matrix transformations and rates of convergence of positive linear operators," Calcolo, vol. 44, no. 3, pp. 159-164, 2007.

[4] O. Duman and E. Erkuş, "Approximation of continuous periodic functions via statistical convergence," Comput. Math. Appl., vol. 52, no. 6-7, pp. 967-974, 2006.

[5] O. Duman, E. Erkuş, and V. Gupta, "Statistical rates on the multivariate approximation theory," Math. Comput. Modelling, vol. 44, no. 9-10, pp. 763-770, 2006.

[6] E. Erkuş, O. Duman, and H. M. Srivastava, "Statistical approximation of certain positive linear operators constructed by means of the Chan-Chyan-Srivastava polynomials," Appl. Math. Comput., vol. 182, no. 1, pp. 213-222, 2006.

[7] H. Fast, "Sur la convergence statistique," Colloquium Math., vol. 2, pp. 241-244, 1951.

[8] J. J. Freedman, A. R.; Sember, "Densities and summability," Pacific J. Math., vol. 95, no. 2, pp. 293-305, 1981.

[9] J. A. Fridy, "On statistical convergence," Analysis, vol. 5, no. 4, pp. 301-313, 1985.

[10] T. Myint-U and L. Debnath, Partial differential equations for scientists and engineers. New York: North-Holland Publishing Co., 1987. 


\section{Author's address}

Esra Erkus-Duman

Gazi University, Faculty of Arts and Sciences, Department of Mathematics, Teknikokullar TR06500, Ankara, Turkey

E-mail address: eduman@gazi.edu.tr 